\title{
Multifractal analysis of the drought area in seven large regions of China from 1961 to 2012
}

\author{
Wei Hou ${ }^{1}$ Guolin Feng ${ }^{2} \cdot$ Pengcheng $\mathrm{Yan}^{3} \cdot$ Shuping $\mathrm{Li}^{3}$
}

Received: 6 September 2015 / Accepted: 9 May 2017 / Published online: 19 May 2017

(c) The Author(s) 2017. This article is an open access publication

\begin{abstract}
Based on a monthly drought index, the Standardized Precipitation Index (SPI), we investigate the multifractality of monthly drought areas in seven major regions in China from 1961 to 2012 using multifractal detrended fluctuation analysis. The results show that multifractality is evident in the monthly time series for all seven regions, but its strength varies between areas. From the numerical results, we further examine the stationarity and persistence of the time series in the seven drought areas. The characteristics of the variance of big and small fluctuations are also analyzed. The characteristics of multifractal spectra are used to distinguish the features of the singularity of all the data, as well as the large and small fluctuations and the spread of the changes of fractal patterns, and so on, in the monthly drought area time series for the different regions. Finally, we investigate the possible source(s) of multifractality in the drought series by random shuffling as well as surrogating the original series for each region.
\end{abstract}

Responsible Editor: C. Simmer.

Wei Hou

houwei@cma.gov.cn

1 National Climate Center, China Meteorological Administration, Beijing 100081, China

2 College of Physical Science and Technology, Yangzhou University, Yangzhou 225002, China

3 College of Atmospheric Sciences, Lanzhou University, Lanzhou 730000, China

\section{Introduction}

Droughts are among the most severe and frequently occurring natural hazards. They have substantial effects on economic, agricultural, ecological, and environmental activity worldwide (Begueria et al. 2010; Li et al. 2013; Vicente-Serrano et al. 2010). Recent studies have indicated an increasing number of global droughts, both observed and modeled (Dai 2013). Since the 1970s, the frequency of extreme droughts has increased in central North China, northeastern China, and the eastern part of northwestern China (Ma and Fu 2006). Despite increasing precipitation, the uneven spatial and temporal distribution of changes in precipitation has resulted in occasional severe droughts in southern China, especially in the southwest (Gao and Yang 2009; Qiu 2010; Song et al. 2003; Zhang et al. 2010a, b, 2011; Zhou et al. 2009).

For the purpose of predicting, monitoring, and assessing the severity of droughts, several numerical drought indices have been developed: the Rainfall Anomaly Index (RAI) (Van-Rooy 1965), Palmer Drought Severity Index (PDSI) (Palmer 1965), Standardized Precipitation Index (SPI), etc. The present study focuses only on the meteorological drought expressed by drought indices. The SPI (Hayes et al. 1999; McKee et al. 1993) is widely used to reveal meteorological droughts and has proved to be a useful tool in estimating the severity and duration of droughts (Bordi et al. 2004; Moreira et al. 2008; Silva et al. 2007). The SPI has also been used in Argentina (Seiler et al. 2002), Canada (Anctil et al. 2002), China (Wu et al. 2005), Europe (Lloyd-Hughes and Saunders 2002), Hungary (Domonkos 2003), India (Chaudhari and Dadhwal 2004), Korea (Min et al. 2003), Spain (Lana et al. 2001), and Turkey (Komuscu 1999) for real-time monitoring or retrospective analysis of droughts. 
Many natural processes, including droughts, may be considered as complex systems. Chaos and fractal theories were one of the important theories to be applied to the analysis of complex systems (Tokinaga 2000; Tsonis 1992; Lorenz 1969a, b). Peng et al. (1994) introduced detrended fluctuation analysis (DFA) to calculate the variance of data at different scales and scaling exponents. Following that, Kantelhardt et al. (2002) extended DFA into multifractal detrended fluctuation analysis (MFDFA) which enables the multifractal behavior of data to be detected, and by studying their shuffled and surrogate time series and comparing them with the results of the original series, the sources of multifractality can be investigated (Jafari et al. 2007; Kimiagar et al. 2009; Lim et al. 2007; Niu et al. 2008; Pedram and Jafari 2008; Telesca et al. 2004). MFDFA has been used to study time series in geophysics (Kantelhardt et al. 2003; Kavasseri and Nagarajan 2005; Koscielny-Bunde et al. 2006), physiology (Dutta 2010; Makowiec et al. 2006, 2011), financial markets (Oswiecimka et al. 2005; Yuan et al. 2009), and the exchange rates of currencies (Norouzzadeh and Rahmani 2006a, b; Oh et al. 2012; Wang et al. 2011a, b). Multifractals describe the dynamic characteristics of systems more carefully and comprehensively, and characterize their properties both locally and globally. This is an important research question, as the evidence of multifractality suggests that multifractal models could be built to allow variables of interest to be forecast, and the prediction study based on multifractality is a hot domain in last decades (de Benicio et al. 2013; Alvarez-Ramirez et al. 2008; Grech and Mazur 2004; Lana et al. 2001, 2010; Martínez et al. 2007, 2010; Sun et al. 2001; Wei and Wang 2008).We can examine the multifractal properties of a time series using MFDFA and any models that contemplate the phenomena should be capable of reproducing the results, such as the relation of intensity to complexity, the sources of multifractality and the relation of small or large scale fluctuations with the increase of intensity. The results of Chen et al. (2004) also showed that some sign sequences of the parameter $1 \mathrm{f}$ could be used to predict the probability of the near future price movements. Wei and Huang (2005) studied the multifractality of the Shanghai Composite Index and proposed volatility measure based on the multifractal spectrum of the intraday price data, the so-called multifractal volatility (MFV). Evaluated by the superior prediction ability (SPA) test, the ARFIMA-MFV model obtained a higher degree of forecasting accuracy than several GARCH-class models.

Since China is one of the countries that suffered severe droughts for years (Weng et al. 2011), and given that a drought is a complex, dissipative and dynamic event, the indications are that the multifractality of droughts should be studied. Some studies have investigated the multifractality of drought data (Jiang et al. 2005; Zhang et al. 2010a, b) and hydrological data related to river flows and rainfall time series. Zhang et al. (2014) given a comparison of detrending methods for fluctuation analysis in hydrology by evaluating the Fourier based detrending method, adaptive detrending algorithm and average detrending technique in eliminating trends in hydrological series, they (Zhang et al. 2008, 2009a, b, 2011, 2014; Yu et al. 2014) analyzed the multifractal properties of rainfall time series and streamflow records in Pearl River basin and the Yangtze River basin of China. The results of these studies provide practical and scientific information in regional flood frequency analysis and water resource management in china. However, there is no such attention has been paid to drought areas. Thus, to better understand the complex behavior of droughts in China, we used MFDFA to examine the multiscaling behavior of drought areas using the SPI on a monthly scale. An analysis of the fluctuations of these particular drought areas, and how these relate to their physical characteristics, contributes to the overall information on the properties of droughts. By applying the MFDFA technique to the monthly time series of the drought areas in seven regions of China over the 50-year period from 1961 to 2012, we identified the probable source of the multifractality in these series by analyzing the randomly shuffled series and the surrogate series corresponding to each of the original series.

This paper is organized as follows: Sect. 2 contains details of the data used in this study and of multifractal detrended fluctuation analysis. In Sects. 3 and 4, we study the multifractal properties and present the results of this analysis. The work is summarized in Sect. 5.

\section{Data and methods}

\subsection{Study area}

China spans many degrees of latitude and has complicated terrain, and therefore the climate varies sharply. Previous studies (Wang et al. 2011a, b; Zhai et al. 2005) were carried out for the whole territory of China subdivided into seven regions, as shown in Fig. 1. These are referred to as Northeastern China (NE), Northern China (N), Northwestern China (NW), Central China (C), Southeastern China (SE), Southwestern China (SW), and Southern China (S). (Note: The analysis excluded Taiwan and Tibet because of inadequate data.) The climatic zones and landscapes of the seven regions differ from each other, making them prone to different levels of water shortage and drought; for example, the arid regions of western and northern China have limited water, and drought is directly related to low precipitation, whereas in some semi-humid or humid regions in eastern China drought is mainly 


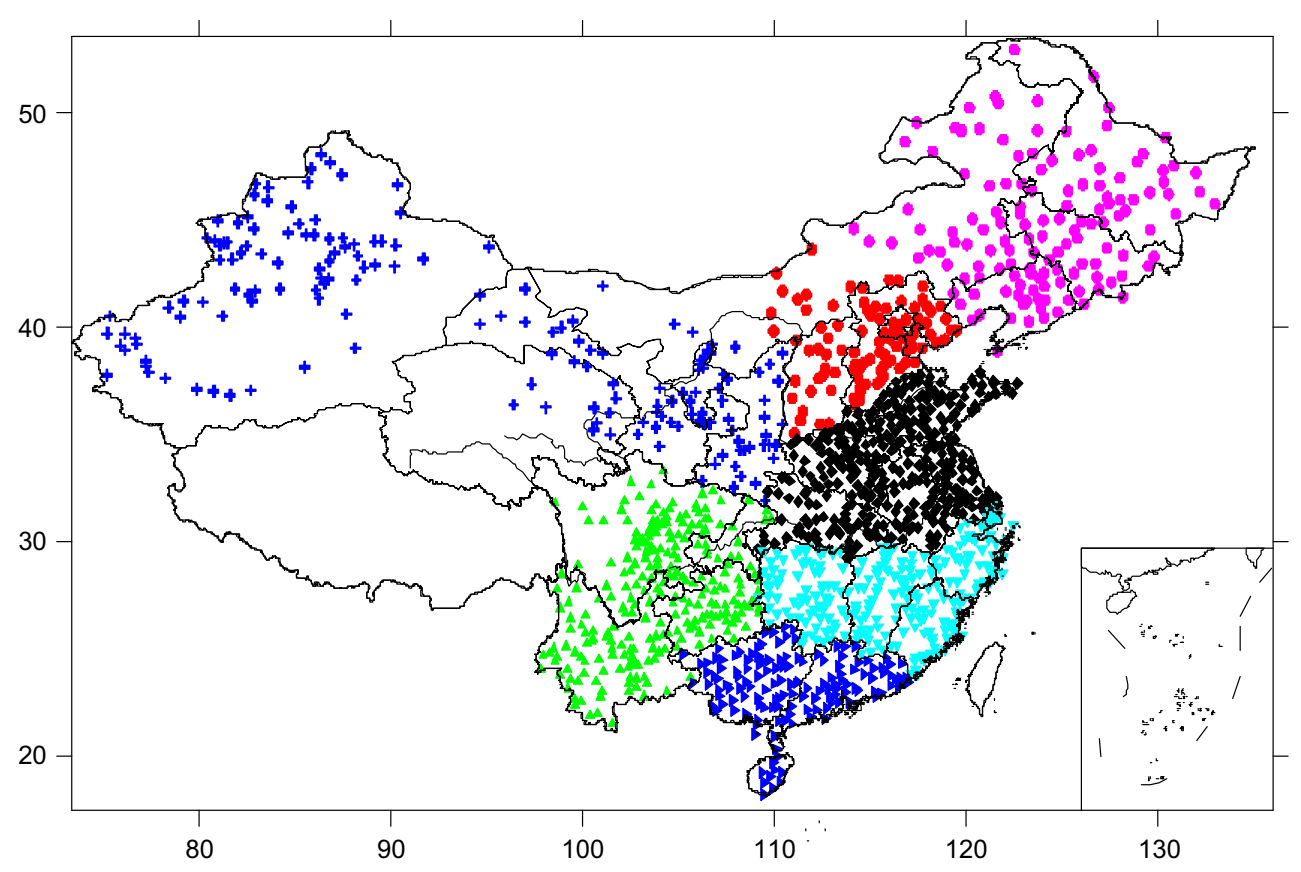

Fig. 1 Location of regions and the spatial distribution of meteorological stations (blue plus symbol NW, red circle $\mathrm{N}$, pink circle NE, black diamond: $\mathrm{C}$, green upward triangle $\mathrm{SW}$, aqua blue downward triangle $\mathrm{SE}$, blue downward triangle $\mathrm{S}$ )

controlled by the East Asian monsoon. Studying droughts in China based on these divisions is beneficial because the different multifractality of each region provides useful information about the dynamic system for efficient prediction.

\subsection{Data sources}

Daily ground-based meteorological observation precipitation data are obtained from the China Meteorological Data Sharing Service System at http://cdc.cma.gov.cn/. Complete data is held by 1283 meteorological stations for the period 1961-2012. Most of these stations are located in eastern China, and they are more evenly distributed there than in the western part of the country: 137 stations in NE, 100 in N, 338 in C, 135 in SE, 146 in S, 173 in NW and 254 stations in SW. The homogeneity of the observed rainfall values used in this study is guaranteed. Station observations collected by the National Meteorological Center of CMA are subject to quality control procedures (Zhai et al. 2005). Currently they constitute the best dataset available for analyzing regional rainfall variations over China. All the observation sites are shown in Fig. 1.

In this study, the 1-month SPIs from 1961 to 2012 were calculated for each Meteorological station following the method of McKee et al. (1993). More details can be found at http://ccc.atmos.colostate.edu/pub/spi.pdf and the SPI calculation procedure can be downloaded from http:// drought.unl.edu/monitor/spi/program/spi_program.htm in southwest China. The meteorological stations with SPI $\leq-1$ are marked as red spots.

To investigate the multifractal properties of drought area, based on the monthly time series and the SPI categories in Table 1, the total number of stations affected by medium, severe and special droughts in each region monthly was calculated for the period being studied. In this paper, The drought area is calculated as follows: suppose there are $N$ meteorological stations $i, i=1,2, \ldots, N$ within a particular region, we first calculated monthly SPI from 1961 to 2012 (a total of 624 months) for all stations $\mathrm{SPI}_{j}^{i}, j=1,2, \ldots, 624$. The drought studied here includes three grades: the medium, severe and severe drought, namely $\mathrm{SPI}_{j}^{i} \leq-1$ according to the classified scales for SPI. We counted the number of stations whose SPI $\leq-1$ each month in this region, and characterized the monthly regional drought area by this statistic. For instance, there were a total of 46 stations whose SPI $\leq-1$ in southwest

Table 1 Classified scales for SPI

\begin{tabular}{lll}
\hline Grade & SPI & Category \\
\hline 1 & $-0.5<$ SPI & No drought \\
2 & $-1.0<$ SPI $\leq-0.5$ & Mild drought \\
3 & $-1.5<$ SPI $\leq-1.0$ & Medium drought \\
4 & $-2.0<$ SPI $\leq-1.5$ & Severe drought \\
5 & SPI $\leq-2.0$ & Special drought \\
\hline
\end{tabular}


China on August 2009, thus the regional drought area was 46 in this month (see Fig. 2a); by the October 2009, there were a total of 91 stations whose SPI $\leq-1$, then the regional drought area was 91 in October 2009 (see Fig. 2b). By the way of analogy, we obtained the monthly regional drought area in each region during 1961-2012 years.

In fact, due to the spatial or geographical characteristics of the stations, the total number of stations suffering from drought characterized the drought area. We then used MFDFA to examine the multifractal behavior of the drought area time series.

\subsection{Multifractal detrended fluctuation analysis (MFDFA)}

The MFDFA of Kantelhardt et al. (2002) takes the fluctuant average of time series in each partition interval as statistical points, and determines a generalized Hurst exponent which depends on the power-law property of the fluctuation function to measure the stationary and non-stationary sequence structures and fluctuation singularity. Recently, the Hurst exponent has been used to detect the complexity and persistence in meteorological or climatic variables (Tatli 2014, 2015). The advantages of this method are that it finds the long-term correlations of non-stationary time series. Computer simulation has demonstrated that the MFDFA method for analyzing multifractality for non-stationary time series was the best of the available methods (Govindan et al. 2007).

The steps in analyzing data characteristics of a drought area based on MFDFA are as follows:
(1) Cumulative deviation of time series $\left\{x_{k}, k=1,2, \ldots, N\right\}$ of monthly drought area data is given by

$Y(i)=\sum_{k=1}^{i}\left[x_{k}-\bar{x}\right]$

where $\bar{x}=\frac{1}{N} \sum_{k=1}^{i} x_{k}$.

(2) Divide sequence $Y(i)$ into $N_{s}$ non-overlapping intervals $v$. Each interval contains the same number of points $s$, where the integral part is $N_{s}=\frac{N}{s}$. Since the length of the sequence is often not an integral multiple of $s$, to disregard this part of the series, the same procedure is repeated starting from the end of the series until $2 N_{s}$ segments are eventually obtained.

(3) Fitting the polynomial of the $v$ th interval by a leastsquares fit of the data for each interval $v\left(v=1,2, \ldots, 2 N_{s}\right)$ is obtained from

$$
\begin{array}{r}
\hat{y}_{v}(i)=\hat{a}_{0}+\hat{a}_{1} i+\cdots+\hat{a}_{m} i^{m}, \\
i=1,2, \ldots, s ; m=1,2, \ldots
\end{array}
$$

The time series removing the trend is denoted by $Y_{s}(i)$, which gives the difference between the original series and fitted values:

$Y_{s}(i)=Y(i)-\hat{y}_{v}(i)$,

where $\hat{y}_{v}(i)$, called an $m$-order MFDFA, is the local trend function of the $v$ th interval, and $m$ is the different fitting order. In MFDFA- $m$ ( $m$ th order MFDFA), trends of order $m$ in the profile (or, equivalently, of order $m-1$ in the original series) are eliminated.
Fig. 2 Spatial distribution of SPI on a August 2009 and b October 2009
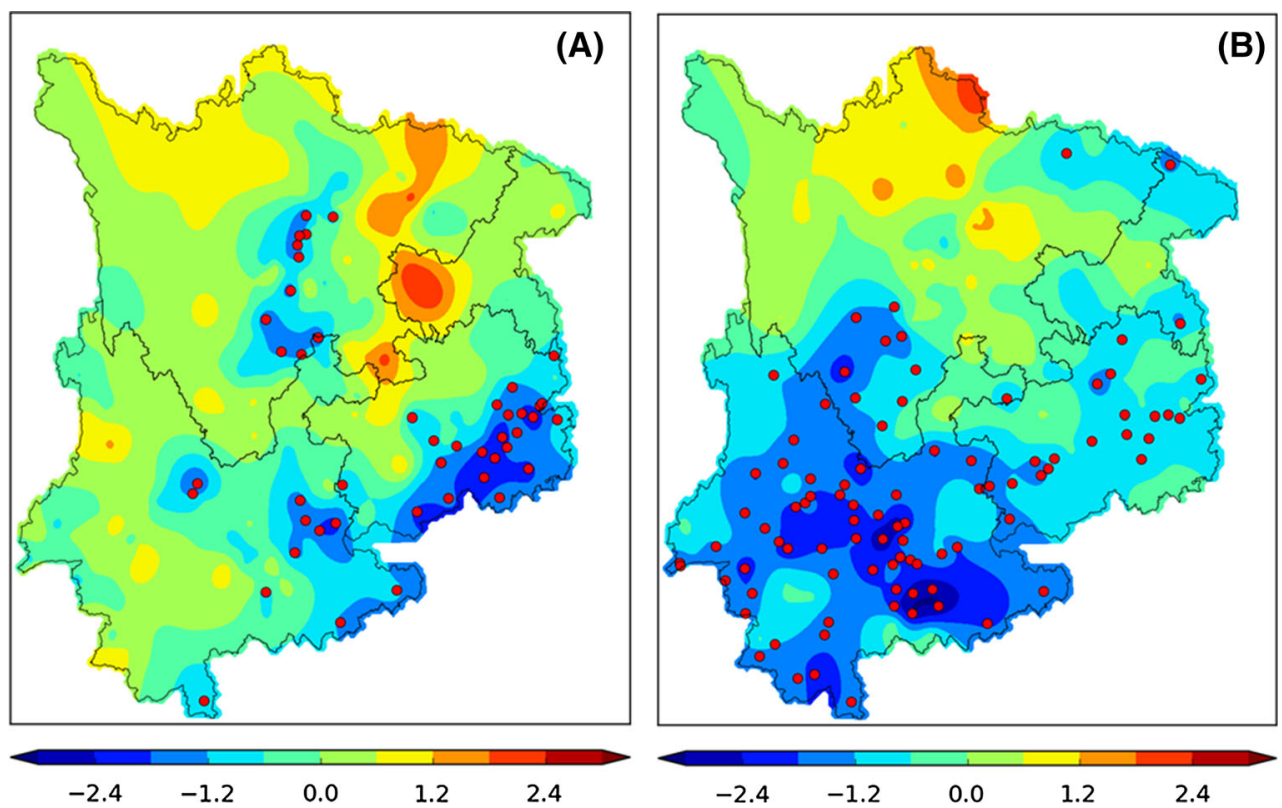
(4) Calculate the local trend for each of the $2 N_{s}$ segments by a least-squares fit of the series. Then determine the variance

$F^{2}(s, v) \equiv \frac{1}{s} \sum_{i=1}^{s}\left\{Y[(v-1) s+i]-\hat{y}_{v}(i)\right\}^{2}$

for each segment $v, v=1,2, \ldots, N_{s}$, and

$F^{2}(s, v) \equiv \frac{1}{s} \sum_{i=1}^{s}\left\{Y\left[N-\left(v-N_{s}\right) s+i\right]-\hat{y}_{v}(i)\right\}^{2}$

for $v=N_{s}, N_{s}+1, \ldots, 2 N_{s}$. Here $y_{v}(i)$ is the fitting polynomial in segment $v$. Different orders have different abilities to eliminate the trend, and the degree of the polynomial may be varied, either to eliminate constant $(m=0)$, linear $(m=1)$, quadratic $(m=2)$ or higher-order trends of the profile. Then $F^{2}(s, v)$ is related to the fitting order.

(5) Average and extract a root for all variances of equallength intervals. Then the $q$-order fluctuation function of the whole sequence is obtained from

$F_{q}(s)=\left\{\frac{1}{2 N_{s}} \sum_{v=1}^{2 N_{s}}\left[F^{2}(s, v)\right]^{q / 2}\right\}^{1 / q}$.

In general, the index variable $q$ takes any real value. For $q=0$, the fluctuation function can be determined from

$F_{q}(s)=\exp \left\{\frac{1}{4 N_{s}} \sum_{v=1}^{2 N_{s}} \ln \left[F^{2}(s, v)\right]\right\}$.

Different $q$ values have different effects on the fluctuation functions. For positive $q$, the segment $v$ with large variance (i.e., large deviation from the corresponding fit) will dominate the average $F_{q}(s)$. Thus, if $q$ is positive, $h(q)$ describes the scaling behavior of the segments with large fluctuations and, in general, large fluctuations are characterized by a smaller scaling exponent $h(q)$ for multifractal time series. For negative $q$, segments $v$ with small variance will dominate the average $F_{q}(s)$. Thus, if $q$ is negative, the scaling exponent $h(q)$ describes the scaling behavior of segments with small fluctuations, usually characterized by a larger scaling exponent $h(q)$.

(6) Determine the scaling exponent of the fluctuation function. Varying the value of $s$ in the range from 15 to $N / 5$, then repeating the procedure described above for various scales $s, F_{q}(s)$ increases with increasing $s$. Then, from $\log -\log$ plots $F_{q}(s)$ vs. $s$ for each value of $q$, the scaling behavior of the fluctuation functions can be determined; thus, if the drought area series $x_{k}$ is correlated to a long-term power-law, $F_{q}(s)$ increases for large values of $s$ as a power-law:

$F_{q}(s) \sim s^{h(q)}$.
Since the scaling behavior of the variances $F^{2}(s, v)$ is identical for all segments $v$, the averaging procedure in Eq. (6) gives an identical scaling behavior for all values of $q$. The family of the exponents $h(q)$ describes the scaling of the $q$ th-order fluctuation function. Multifractality refers to the scaling property of a time series that needs to be represented by an array of scaling exponents, but not by any single one. The multifractality of a time series may be identified by examining the dependence of $h(q)$ on highand low-order moments. If the time series is monofractal, then $h(q)$ is independent of $q$, but if the series is multifractal, then $h(q)$ depends on $q$, thereby characterizing a single form of self-similarity over time; $h(q)$ depends significantly on $q$ only if large and small fluctuations scale differently. Since richer multifractality obviously corresponds to higher variability of $h(q)$, the degree of multifractality is quantified by

$\Delta h=h\left(q_{\min }\right)-h\left(q_{\max }\right)$.

As large fluctuations are characterized by smaller scaling exponents $h(q)$ than small fluctuations, $h(q)$ for $q<0$ are larger than those for $q>0$, and $\Delta h$ is positively defined.

For $q=2$, it is seen that Eqs. (4) and (6) are the same, and the standard DFA procedure is retrieved, so that at this point DFA is a special case of MFDFA. In general, the exponent $h(q)$ will depend on $q$. For stationary time series, $h(2)$ is the well-defined Hurst exponent $H$. Thus, $h(q)$ is called the generalized Hurst exponent. For a stationary time series (e.g., fractional Gaussian noise), the profile defined in Eq. (1) will be a fractional Brownian motion. Thus, $0<h(q=2)<1$ for these processes, and $h(q=2)$ is identical to the Hurst parameter $H$. On the other hand, if the original signal is a fractional Brownian motion, the profile will be the sum of fractional Brownian motions, so $h(q=2)>1$. In this case the relationship between the exponent $h(q=2)$ and $H$ is $H=h(q=2)-1$. Further details may be found in Movahed et al. (2006).

The above equality can be also expressed as $F_{q}(s) \sim A s^{h(q)}$. Taking the logarithm of both sides of the equation,

$\ln \left(F_{q}(s)\right)=\ln A+h(q) \ln (s)$.

A corresponding fluctuation function value $F_{q}(s)$ can be obtained for each partition length $s$. Different $F_{q}(s)$ are obtained using different constant $s$. From least-squares linear regression for the above equality, the estimated gradient is the $q$-order generalized Hurst exponent $h(q)$.

(7) The $h(q)$ obtained using MFDFA is related to the Rényi exponent $\tau(q)$; that is,

$\tau(q)=q h(q)-1$. 
(8) The multifractal spectrum $f(\alpha)$, which describes multifractal time series, is obtained from

$\alpha=h(q)+q h^{\prime}(q)$,

where $\alpha$ is the Hölder exponent or singularity strength, and

$f(\alpha)=q[\alpha-h(q)]+1$.

The shape and extent of the singularity spectrum $f(\alpha)$ curve contains significant information about the distribution characteristics of the examined dataset, and describes the singularity content of the time series. In the generalized binomial multifractal model, the strength of the multifractality of a time series is characterized by the difference between the maximum and minimum values of $\alpha$, $\Delta \alpha=\alpha_{\max }-\alpha_{\min }$. It should be noted that this parameter is identical to the width of the singularity spectrum $f(\alpha)$ at $f=0$. A wider singularity spectrum indicates a richer multifractality (Norouzzadeh and Rahmani 2006a, b).

\section{Multifractal spectrum analysis}

We analyzed the multifractal behavior of the drought area time series in each region by implementing the MFDFA technique using a second-order detrending polynomial, $m=2$. The drought area time series was then divided into $N_{s}$ non-overlapping bins. The value of $s$ was chosen in the range 15 to $N / 5$ in steps of 1 , for $N=624$. We restricted the moment $q$ to the range $[-10,10]$ with steps equal to 1 . The results obtained for only one of these are shown in Figs. 3 and 4 .

The MFDFA results for the monthly drought area time series of the seven regions in this study are shown in Fig. 3a. It is seen that $h(q)$ are decreasing functions that exhibit a dependence on $q$, which is typical of multifractal behavior. Thus, Fig. 3a reveals the presence of multifractal behavior in the monthly drought area time series for the seven regions in China.

To evaluate and compare the multifractality degree of the time series in the study, the range $\Delta h$ of the $h(q)$ was calculated. A greater degree of multifractality $\Delta h$ corresponds to a higher rate of decrease- that is, a steeper $h(q)$ curve, in which the variability in the distribution of high and low fluctuations is increased and the temporal distribution of the drought area is more complex and heterogeneous. Conversely, a smaller range of $\Delta h$ indicates a low rate of decrease-that is, a gentle $h(q)$ curve with smaller variation in the distribution of high and low fluctuations, indicating a more regular and homogeneous temporal distribution of the drought areas.

Figure $3 \mathrm{~b}$ shows the $\Delta h$ of the drought area for seven regions. It is clear that $\Delta h$ for central and eastern China is

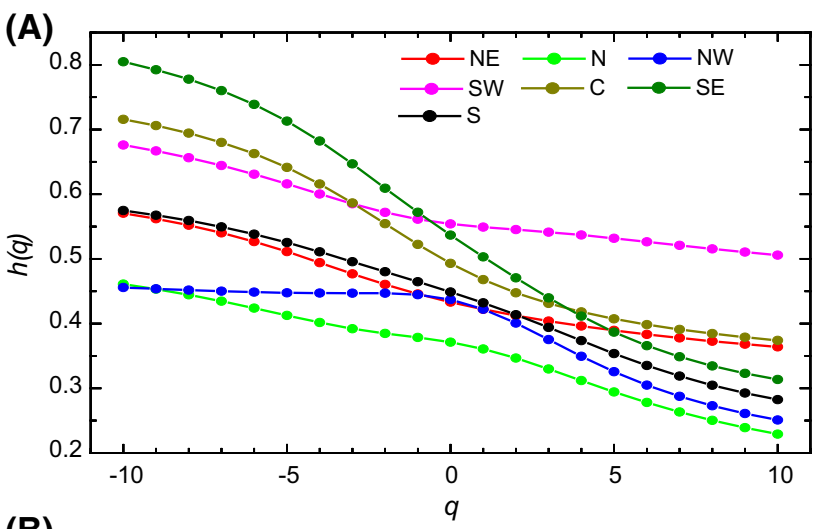

(B)
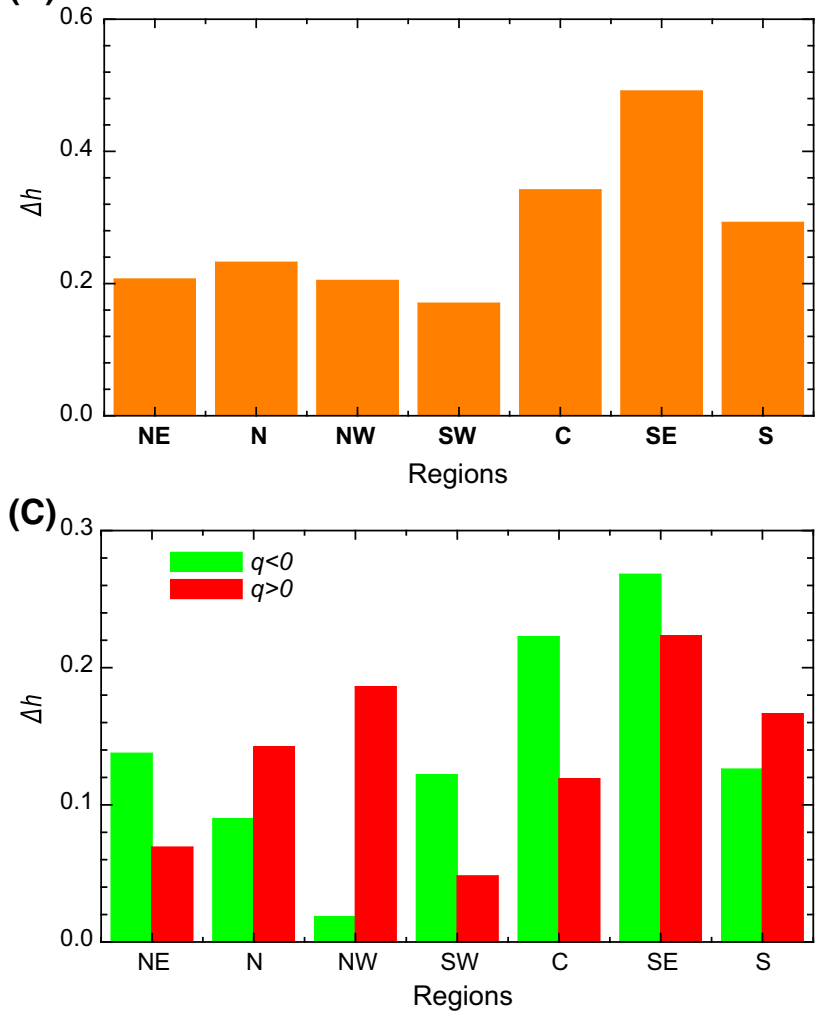

Fig. 3 a $h(q)$ curves; b whole $\Delta h$; and $\mathbf{c}$ sectional $\Delta h$ of monthly drought area for seven regions in China

greater than in regions in western and northern China. This indicates that the variation in the distribution of fluctuations of the drought areas in central and eastern China is greater than in western and northern China. The largest variation is seen in SE $(\Delta h=0.49)$; that is, the heterogeneity and complexity of the temporal distribution of drought areas in SE is also the most marked of all seven regions. The second-largest variation is seen in $\mathrm{C}$ $(\Delta h=0.34)$. The least variation occurred in SW, where the smallest $\Delta h$ values are reached $(\Delta h=0.17)$, indicating that the temporal distribution of drought areas in SW is the most homogeneous and regular of the seven regions. The values of $\Delta h$ in $\mathrm{S}, \mathrm{N}, \mathrm{NE}$ and $\mathrm{NW}$ are almost identical 
(ranging from 0.29 to 0.20 ), indicating that the temporal distribution of droughts is more regular and homogeneous than in SE and C.

In Fig. 3a, it is notable that $h(q)$ values in SW are all greater than 0.5 . This indicates that the drought areas in SW have an obvious long memory, with persistent fluctuations in both large and small drought areas. Conversely, the $h(q)$ in $\mathrm{N}$ and $\mathrm{NW}$ are all $<0.5$, indicating shortmemory drought areas in which the fluctuations of the drought areas are intermittent, or non-persistent. On the whole, the persistence of small fluctuations of drought areas is weaker, in descending order, in the SE, C, SW, S, and $\mathrm{NE}$ regions, and the intermittent nature of large fluctuations of drought areas is stronger, in ascending order, in the $\mathrm{C}, \mathrm{NE}, \mathrm{SE}, \mathrm{S}, \mathrm{NW}$, and $\mathrm{N}$ regions. The large discrepancy between SW and the other regions is in terms of positive $q$, indicating that it is mainly related to the scaling properties of the large fluctuations.

Changes of $h(q)$ mainly depend on the variation of small fluctuations if $q<0$, and mainly depend on the variation of large fluctuations if $q>0$. Figure $3 \mathrm{c}$ shows that, for $\mathrm{NE}$, $\mathrm{SW}$, and $\mathrm{C}$, the ranges $\Delta h_{q<0}$ are larger than $\Delta h_{q>0}$, which indicates that the variation of small fluctuations is greater than the variation of large fluctuations in those three regions, since the sparsely populated time domains are more heterogeneous and complex than those densely clustered on the time axis. In $\mathrm{N}$ and $\mathrm{NW}, \Delta h_{q>0}$ are larger than $\Delta h_{q<0}$, indicating that large fluctuations vary more than small fluctuations in the two regions, where the densely clustered time domains are more heterogeneous and complex than the more sparsely populated time axis. For $\mathrm{SE}$ and $\mathrm{S}, \Delta h_{q>0}$ are almost identical to $\Delta h_{q<0}$, which implies that the variation in large fluctuations is almost the same as the variation in small fluctuations, and the densely clustered time domains are almost the same as the sparsely populated domains.

The scaling exponent $h(2)=0.54$ exceeds 0.5 for the $\mathrm{SW}$ region. This indicates that the drought area was stationary and long-term correlated or persistent; however, the $h(2)$ are all less than 0.5 for the other six regions, which indicates that their drought areas tended to be anti-persistence (or mean-reverting process) and tended to be nonstationary and short-term correlated or intermittent. The strongest short-term correlation is found in N, shown by its smallest scaling exponent $h(2)=0.35$, whereas NW, NE, $\mathrm{S}, \mathrm{C}$, and SE have a weaker short-term correlation with $h(2)=0.40,0.41,0.42,0.45$, and 0.47 , respectively.

The $h(2)$ clearly exceeding 0.5 suggests persistence, in other words, forthcoming predictions of elements will be strongly governed by trends on the preceding elements. On the contrary, values of $h(2)$ lowering 0.5 will suggest antipersistence. In this case, a good estimation of new elements will take into account an average of previous elements. Due to the long-term correlations in the drought area time series, large values are rather followed by large values and small values are rather followed by small values, leading to periods with fewer drought events and to a clustering of more drought events on the other hand. A short-term correlations in the drought area time series indicate that an up value will most likely be followed by a down value and vice versa, which means that future values have a tendency to return to a long-term mean. The stronger short-term correlation switch their sign more frequently and a random process does (Feder 1988; Kantelhardt 2008; Mensia et al. 2017; Mali et al. 2017). Finally, $h(2)$ close to 0.5 evidences series modeled by pure randomness. Consequently, forthcoming elements could reach any value within the range of the sample data (Turcotte 1997; Hurst et al. 1965).The predictability of the drought area in SW and $\mathrm{N}$ was higher than in other regions. In descending order, the predictability of the drought areas was increasingly weak in NW, NE, S, C, and SE (toward 0.5).

The multifractal spectra adequately characterize the multifractal nature of drought areas, which enables the fluctuations of areas and the previously defined time scales to be described in detail. For a multifractal time series, the shape of the singularity spectrum resembles a
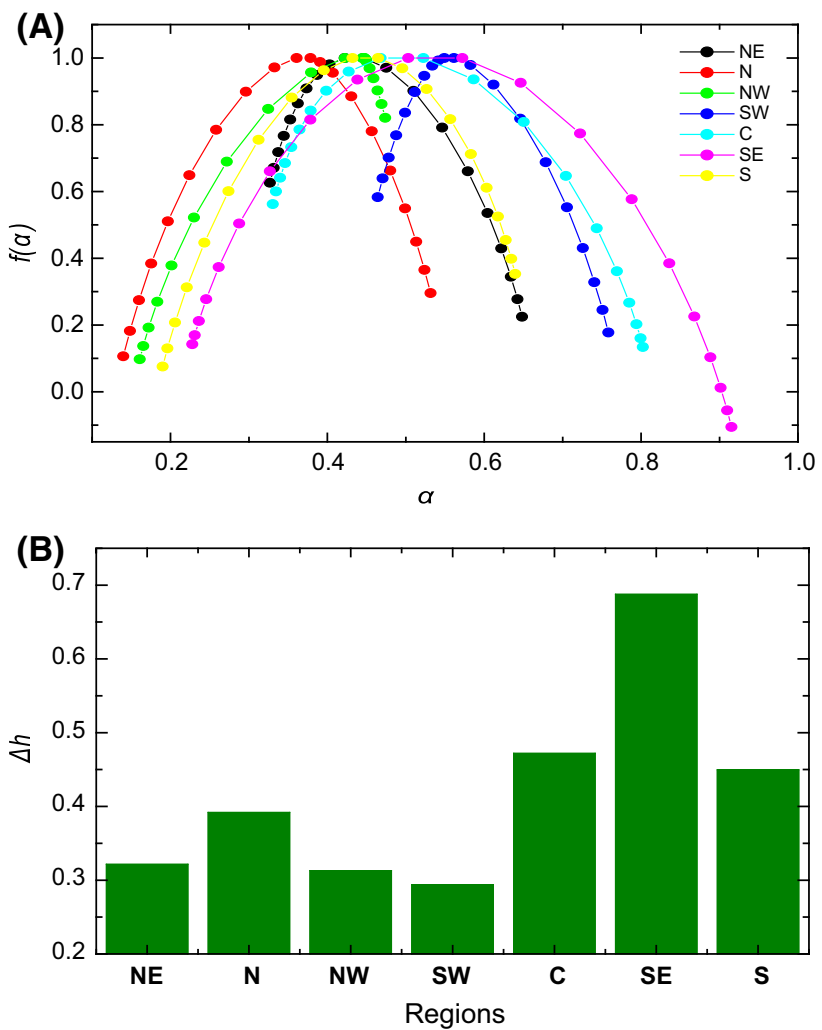

Fig. 4 a Multifractal spectra; $\mathbf{b}$ width of monthly drought areas for the seven regions 
wide inverted parabola whose left- and right-hand wings refer to positive and negative $q$, respectively. For pure multifractals, the value of $\alpha$ decreases with increased fluctuation.

The multifractal spectrum curves $f(\alpha)$ of the studied elements were calculated by the moment method (Halsey et al. 1986). In all cases, the multifractal spectra (see Fig. 4a) are all continuous and asymmetrical with convex parabolas, which confirms that the drought area time series for the seven regions are multifractal, but to a varying degree, since the curves are all different. All of them reach the maximum $f(\alpha)$ value of 1 , relating to the single dimension of the studied variables. When the spectra are compared, we observe that their shapes are different; consequently, all of the studied datasets are subject to different high and low fluctuations.

Several useful indices were used in this work: $\Delta \alpha=\alpha_{\max }-\alpha_{\min }, \quad \Delta \alpha_{\mathrm{L}}=\alpha_{0}-\alpha_{\min }, \quad \Delta \alpha_{\mathrm{R}}=\alpha_{\max }-\alpha_{0}$, $R=\frac{\Delta \alpha_{L}-\Delta \alpha_{R}}{\Delta \alpha_{L}+\Delta \alpha_{R}}, \Delta f(\alpha)=f\left(\alpha_{\text {mix }}\right)-f\left(\alpha_{\max }\right)$, where $\alpha_{0}$ is the particular value of $\alpha$ corresponding to $q=0 ; \alpha_{\min }$ and $\alpha_{\max }$ are the minimum and maximum values of $\alpha$, respectively. The width $\Delta \alpha$ of a multifractal spectrum provides a measure of multifractality: a larger range of $\Delta \alpha$ indicates a wider multifractal spectra, implying a higher degree of multifractality. A larger or smaller multifractal signal (corresponding to a larger or smaller $\Delta \alpha$ ) implies a greater or lesser heterogeneous signal. A signal is heterogeneous if it is characterized by sudden bursts of high frequency, intermittencies and/or irregularities. $\Delta \alpha_{L}$ and $\Delta \alpha_{R}$ are, respectively, the left- and right-hand branches of the multifractal spectrum curve; their values describe the distribution patterns of high and low fluctuations, respectively (Agterberg 2001; Evertsz and Mandelbrot 1992). The asymmetry index $(R)$, which ranges from -1 to 1 , quantifies the deviations of the multifractal spectrum curve (Xie and Bao 2004): $R>0$ suggests a left-hand deviation of the multifractal spectrum, likely to have resulted from some degree of local high fluctuations; $R<0$ suggests a righthand deviation with local low fluctuations; and $R=0$ represents a symmetrical multifractal spectrum. $\Delta f(\alpha)$ is the difference between the maximum and minimum values of $f(\alpha)$.

Table 2 Multifractal parameters of the studied data
The result of the $\Delta \alpha$ values for different regions is shown in Fig. 4b and Table 2. Similar to $h(q)$, the results show that the regions in central and eastern China have stronger multifractal fluctuations (larger $\Delta \alpha$ ); that is, greater variation in the distribution of fluctuations and more heterogeneous temporal distribution of drought areas, than the regions in western and northern China. The highest $\Delta \alpha$ for SE suggests that this region has the greatest irregularity and multifractality, and therefore the singularity of its drought area time series is the greatest, which indicates that the changes in the drought area in this region are more extreme than elsewhere and the prediction was the most difficult in SE. The $\Delta \alpha$ of $\mathrm{C}$ and $\mathrm{S}$ are similar, both higher than in the other four regions and the prediction was also very difficult in C and S. The $\Delta \alpha$ of SW is the smallest, meaning that changes in the drought area in this region are smaller than in the other regions and the prediction was the easiest relatively in SW; and the $\Delta \alpha$ of NE, N, and NW are also similar and the drought area in these regions also has a low predictability.

Furthermore, the multifractal spectrum of SE is symmetrical $(R \approx 0)$, and $\Delta \alpha_{\mathrm{L}} \approx \Delta \alpha_{\mathrm{R}}$, that is, the singularity of the large and small fluctuations is identical. The multifractal spectrum of N, NW, and S is left-deviant. Of these, left-deviation is most significant and $\Delta \alpha_{L} \gg \Delta \alpha_{R}$ in NW with the highest the values of $\Delta \alpha_{\mathrm{L}}$, which implies that the singularity with the high values is greatest, and it is far greater in magnitude than low-value cases; it also has the largest local high fluctuations in drought clusters spatially. In $\mathrm{N}$ and $\mathrm{S}$ it is almost the same, with $\Delta \alpha_{\mathrm{L}}$ slightly larger than $\Delta \alpha_{R}$, but there is no significant difference of singularity between the high and the low values in those regions, with droughts showing only slightly higher spatial clusters locally.

The multifractal spectra of SW, NE, and C, in that sequence, are right-deviant - that is, the singularity of the low values is larger than the high values, and local droughts in those regions have been more sparse spatially.

The difference $\Delta f(\alpha)$ between maximum and minimum values of the singularity provides an estimate of the spread in changes in fractal patterns. Since $\Delta f(\alpha)$ denotes the frequency ratio of the largest to the smallest fluctuation,

\begin{tabular}{lllllllllrr}
\hline & $\alpha_{\min }$ & $\alpha_{\max }$ & $\alpha_{0}$ & $\Delta \alpha_{\mathrm{L}}$ & $\Delta \alpha_{\mathrm{R}}$ & $\Delta \alpha$ & $R$ & $f\left(\alpha_{\min }\right)$ & $f\left(\alpha_{\max }\right)$ & $\Delta f(\alpha)$ \\
\hline $\mathrm{NE}$ & 0.327 & 0.648 & 0.446 & 0.119 & 0.202 & 0.321 & -0.259 & 0.626 & 0.225 & 0.401 \\
$\mathrm{~N}$ & 0.140 & 0.532 & 0.379 & 0.239 & 0.153 & 0.392 & 0.2190 & 0.106 & 0.295 & -0.189 \\
$\mathrm{NW}$ & 0.161 & 0.474 & 0.445 & 0.284 & 0.029 & 0.313 & 0.814 & 0.097 & 0.820 & -0.723 \\
$\mathrm{SW}$ & 0.464 & 0.758 & 0.561 & 0.097 & 0.197 & 0.294 & -0.339 & 0.583 & 0.178 & 0.405 \\
$\mathrm{C}$ & 0.330 & 0.802 & 0.522 & 0.192 & 0.280 & 0.472 & -0.186 & 0.562 & 0.134 & 0.428 \\
$\mathrm{SE}$ & 0.228 & 0.915 & 0.572 & 0.344 & 0.343 & 0.687 & 0.002 & 0.142 & -0.105 & 0.247 \\
$\mathrm{~S}$ & 0.190 & 0.640 & 0.465 & 0.275 & 0.175 & 0.450 & 0.222 & 0.075 & 0.353 & -0.278 \\
\hline
\end{tabular}


$\Delta f(\alpha)>0$ means that the largest fluctuations are more frequent than smallest fluctuations, while $\Delta f(\alpha)<0$ is the reverse. It is apparent that $\Delta f(\alpha)$ are positive in NE, SW, C, and SE, which reveals that the largest fluctuations of these drought areas are more frequent than the smallest fluctuation areas. The size of this characteristic is much the same in NE, SW, and C, but it is weaker in SE. In N, NW, and S, $\Delta f(\alpha)$ are negative- that is, the smallest fluctuations of drought areas are more frequent than the largest fluctuations, especially for NW, but it is weaker in $\mathrm{N}$ than in $\mathrm{S}$.

\section{Sources of multifractality}

Generally, there are two types of source of multifractality in time series. One is due to different long-term temporal correlations for small and large fluctuations; the other is due to the fat-tailed distributions of variations (Matia et al. 2003). To investigate the dynamic causes of multifractality in drought areas, both methods were used in this study. The shuffling procedure destroys any temporal correlations in the data, but the distributions remain exactly the same. In order to quantify the influence of the fat-tail distribution, surrogate time series were generated from the original by randomizing their phases in Fourier space, so that the surrogate series are Gaussian. If the multifractality is derived from temporal correlations, the generalized Hurst exponent $h(q)$ obtained by shuffling the data should have a constant value of 0.5 ; then, if temporal correlation was the only reason for the multifractal features, after the series is phase-randomized, $h(q)$ should be independent of $q$; but if both sources are the reasons for the multifractal features, the multifractality should remain but its strength should be diminished.

The shuffling procedure consists of the following steps (Matia et al. 2003): first, generate $(m, n)$ pairs of random integer numbers which satisfy $m, n \leq N$, where $N$ is the length of the time series to be shuffled; then interchange entries $m$ and $n$ in the time series. It is critical to ensure that the ordering of entries in the time series is fully shuffled, such that the long-term or short-term memories, if any, are destroyed. The shuffling is repeated with different random seeds to avoid systematic errors in the random number generators. The algorithm of phase randomization (Norouzzadeh et al. 2007; Small and Tse 2003) is determined by firstly taking the discrete Fourier transform of the time series, then shuffling the phases of the complex conjugate pairs (noting that the phases of complex numbers must be shuffled pairwise to preserve the realness of the inverse Fourier transformation) and finally, taking the inverse Fourier transformation. Ten different realizations of the shuffled and surrogate time series associated to the monthly drought area were generated in this way to reduce statistical errors.
Table 3 Generalized Hurst exponents and width of multifractal spectrum

\begin{tabular}{|c|c|c|c|}
\hline Region & Original & Shuffled & Surrogate \\
\hline \multicolumn{4}{|l|}{$\mathrm{NE}$} \\
\hline$h(-10)$ & 0.571 & 0.663 & 0.542 \\
\hline$h(10)$ & 0.364 & 0.469 & 0.387 \\
\hline$h(2)$ & 0.412 & 0.503 & 0.436 \\
\hline$\Delta h$ & 0.207 & 0.194 & 0.155 \\
\hline$\Delta \alpha$ & 0.321 & 0.317 & 0.261 \\
\hline \multicolumn{4}{|l|}{$\mathrm{N}$} \\
\hline$h(-10)$ & 0.461 & 0.682 & 0.509 \\
\hline$h(10)$ & 0.229 & 0.454 & 0.308 \\
\hline$h(2)$ & 0.347 & 0.504 & 0.374 \\
\hline$\Delta h$ & 0.232 & 0.228 & 0.201 \\
\hline$\Delta \alpha$ & 0.392 & 0.360 & 0.322 \\
\hline \multicolumn{4}{|l|}{ NW } \\
\hline$h(-10)$ & 0.456 & 0.582 & 0.541 \\
\hline$h(10)$ & 0.251 & 0.422 & 0.370 \\
\hline$h(2)$ & 0.400 & 0.493 & 0.423 \\
\hline$\Delta h$ & 0.205 & 0.160 & 0.171 \\
\hline$\Delta \alpha$ & 0.313 & 0.265 & 0.276 \\
\hline \multicolumn{4}{|l|}{ SW } \\
\hline$h(-10)$ & 0.676 & 0.573 & 0.647 \\
\hline$h(10)$ & 0.506 & 0.444 & 0.505 \\
\hline$h(2)$ & 0.545 & 0.500 & 0.561 \\
\hline$\Delta h$ & 0.170 & 0.129 & 0.143 \\
\hline$\Delta \alpha$ & 0.294 & 0.221 & 0.245 \\
\hline \multicolumn{4}{|l|}{$\mathrm{C}$} \\
\hline$h(-10)$ & 0.716 & 0.705 & 0.618 \\
\hline$h(10)$ & 0.374 & 0.444 & 0.403 \\
\hline$h(2)$ & 0.448 & 0.507 & 0.488 \\
\hline$\Delta h$ & 0.342 & 0.231 & 0.215 \\
\hline$\Delta \alpha$ & 0.472 & 0.399 & 0.337 \\
\hline \multicolumn{4}{|l|}{ SE } \\
\hline$h(-10)$ & 0.805 & 0.686 & 0.615 \\
\hline$h(10)$ & 0.313 & 0.464 & 0.455 \\
\hline$h(2)$ & 0.471 & 0.505 & 0.521 \\
\hline$\Delta h$ & 0.491 & 0.222 & 0.161 \\
\hline$\Delta \alpha$ & 0.687 & 0.341 & 0.270 \\
\hline \multicolumn{4}{|l|}{ S } \\
\hline$h(-10)$ & 0.575 & 0.656 & 0.544 \\
\hline$h(10)$ & 0.282 & 0.418 & 0.391 \\
\hline$h(2)$ & 0.414 & 0.494 & 0.441 \\
\hline$\Delta h$ & 0.292 & 0.238 & 0.153 \\
\hline$\Delta \alpha$ & 0.450 & 0.365 & 0.255 \\
\hline
\end{tabular}

From the results for the shuffled and surrogate cases listed in Table 3, we found that the spectrum widths for all seven regions became slightly narrower after the phase randomization and shuffling procedures. At the same time, 
for NW and SW, $\Delta \alpha$ and $\Delta h$ changed less between the surrogate data and the original data than between the shuffled data and the original data, so that temporal correlations contributed more to multifractality formation in those two regions; but the changes were larger after phase randomization than after shuffling for the other five regions, Thus, non-Gaussian distribution contributed more to multifractality formation in those regions.

In particular, when $q=2$ for the shuffled returns, the Hurst exponents of all seven regions were around 0.5 (see Table 3). These results clearly indicate that the shuffled series obeyed random walk.

\section{Summary}

In this work, we investigated the fractal properties of the drought area time series obtained from seven large regions in China from 1961 to 2012, using the MFDFA method, which allows the non-linearity and complexity of drought dynamics to be determined. The $q$-dependence of the generalized Hurst $h(q)$ and multifractal spectrum indicated that the drought area time series from all seven regions demonstrated multifractal behavior. The seven subregions are referred to as North Eastern China (NE), Northern China (N), North Western China (NW), Central China (C), South Eastern China (SE), South Western China (SW), and Southern China (S).

The result of $h(q)$ shows that the drought area in SW has an obvious long memory, with persistent large and small fluctuations in the drought area. The drought areas in $\mathrm{N}$ and NW have a short memory, such that the fluctuations of the drought areas are intermittent. In addition, the persistence of small fluctuations of drought areas in SE, C, SW, S, and NE regions was increasingly weak, in that order. The nonpersistence of the large fluctuations of drought areas in C, $\mathrm{NE}, \mathrm{SE}, \mathrm{S}, \mathrm{NW}$, and $\mathrm{N}$ regions was increasingly strong, in that order.

The results of $h(2)$ show that the drought area in SW was stationary and long-term correlated or persistent, but in the other six regions the drought area tended to be nonstationary and short-term correlated or anti-persistent.

According to $\Delta h$ and $\Delta \alpha$ for each region, the variability in the distribution of fluctuations (evidenced by the heterogeneity and complexity of the temporal distributions) of the drought areas is increasingly weak in SE, C, S, N, $\mathrm{NE}, \mathrm{NW}$, and SW, in that order. Conversely, in ascending order, the homogeneity and regularity of the temporal distribution of the drought areas were increasingly strong in SW, NW, NE, N, S, C, and SE.

From the results of $\Delta h_{q<0}$ and $\Delta h_{q>0}$, it was found that the variability of the small fluctuations was larger than the large fluctuations in three of the regions, and the sparsely populated time domains were more heterogeneous and complex than the dense clusters on the time axis in NE, SW, and C. In N and NW, the variations in large fluctuations was greater than variations in small fluctuations in three regions, and the densely clustered time domains were more heterogeneous and complex than the sparsely populated zones on the time axis. In SE and $\mathrm{S}$, the variation of the large fluctuations was identical to the variation of the small fluctuations, and the densely clustered time domains were almost identical to the sparsely populated domains.

In SE, the singularity of large and small fluctuations in the drought area was identical. In NW, the singularity in the high values is greatest, and far higher in the case of the low values, and had the highest local high fluctuations (drought cluster) spatially. In $\mathrm{N}$ and $\mathrm{S}$ no significant difference of singularity was detected between the high and low values in the drought area, with slightly higher local spatial clusters. In SW, NE, and C, in that order, the singularity of the low values was larger than for the high values with more sparse local drought areas spatially.

In NE, SW, C, and SE, the largest fluctuations of drought areas occurred more frequently than the smallest fluctuations in those regions. In N, NW, and S, the smallest fluctuations of drought areas were more frequent than the largest fluctuations, especially in NW.

By comparing the results of the original drought area time series with those of the shuffled and surrogate series, we found that for NW and SW, temporal correlations contributed most to multifractality formation; for the other five regions, the multifractality due to the broad PDF made a greater contribution than correlation. In NW and SE, the contributions from non-Gaussian distribution and temporal correlation are roughly equivalent.

China is located in the East Asian monsoon region. Rainfalls are highly concentrated in rainy seasons, frequently resulting in floods and droughts when they are quite anomalous. The multifractal features of droughts of each regions of China are associated with the seasonal, interannual variation, interdecadal, and decadal variations of southerly monsoon flow in eastern and southern China and westerly flow in northwestern China. Such variation in the distribution of fluctuations of the drought areas in eastern China is greater than in western and northern China, as the onset and the seasonal northward advance of the East Asian monsoon. In East China, approximately $110^{\circ} \mathrm{E}$, the rainfall gradually extends northward with the season and is influenced by southeasterly monsoon flow coming from the South China Sea and the tropical west Pacific. Along the longitude of the East China Sea, the southerly monsoon rainfall is mixed with the westerly precipitation in the Northeast China region (Qian et al. 2009). The variability of strength and onset and termination 
times of this large-scale circulation may affect convective activities that determine the intensity and frequency of rainfall events. Moreover, the single-peak pattern and the multi-peak pattern with an obvious break periods between two adjacent peaks can be observed in the climatic pentad rainfall series in China. The single-peak pattern reflects the annual cycle of rainfalls, while the multi-peak pattern shows obvious effect of the climatic intraseasonal oscillations. The single-peak pattern is widely distributed in most regions of China, including the Central China, Northeast China, North China, Northwest China, with the major rainy season representing the main character of annual cycle of rainfalls. However, annual rainfalls also show significant multi-peak pattern in Southern China, Southwestern China, and the middle and Southeastern China, where the spring rainfalls and autumn rainfalls being much obvious except for the major rainy season during summer. Thus, the rainy seasons in these regions consist of the spring rainfalls, the major rainy season, and the autumn rainfalls in an annual cycle (Qian and Lin 2005; Ding and Wang 2008).

This work contributes to a better understanding of the scaling laws of drought in different regions in China, and may be helpful in issues of forecasting, model assessment, and characterization of arid-meteorological variation.

Acknowledgements This work is financially supported by the National Natural Science Foundation of China (Grant Nos. 41305056, 41675092) and the key Special Scientific Research Fund of Meteorological Public Welfare Profession of China (Grant No. GYHY201506001).

Open Access This article is distributed under the terms of the Creative Commons Attribution 4.0 International License (http://crea tivecommons.org/licenses/by/4.0/), which permits unrestricted use, distribution, and reproduction in any medium, provided you give appropriate credit to the original author(s) and the source, provide a link to the Creative Commons license, and indicate if changes were made.

\section{References}

Agterberg FP (2001) Multifractal simulation of geochemical map patterns. In: Merriam DF, Davis JC (eds) Geologic modeling and simulation: sedimentary systems. Kluwer, New York, pp 327-346

Alvarez-Ramirez J, Alvarez J, Rodriguez E (2008) Short term predictability of crude oil markets: a detrended fluctuation analysis approach. Energy Econ 30:2645-2656

Anctil F, Larouche W, Viau AA, Parent LE (2002) Exploration of the standardized precipitation index (SPI) with regional analysis. Can J Soil Sci 82:527-538

Begueria S, Vicente-Serrano SM, Angulo-Martinez M (2010) A multiscalar global drought dataset: the SPEIBASE A new gridded product for the analysis of drought variability and impacts. Bull Am Meteorol Soc 91:1351-1354

Bordi I, Fraedrich K, Gerstengarbe FW, Werner PC, Sutera A (2004) Potential predictability of dry and wet periods: sicily and ElbeBasin (Germany). Theor Appl Climatol 77:125-138
Chaudhari KN, Dadhwal VK (2004) Assessment of impact of drought-2002 on the production of major kharif and rabi crops using standardized precipitation index. J Agrometeorol 6:10-15

Chen H, Sun X, Wu Z, Wang B (2004) Enlightenment from various conditional probabilities about the Hang Seng index in the Hong Kong stock market. Physica A 335:183-196

Dai AG (2013) Increasing drought under global warming in observations and models. Nat Clim Change 3:52-58

de Benicio RB, Stošić T, de Figueirêdo PH, Stošić BD (2013) Multifractal behavior of wild-land and forest fire time series in Brazil. Physica A 392:6367-6374

Ding YH, Wang ZY (2008) A study of rainy seasons in China. Meteorol Atmos Phys 100:121-138

Domonkos P (2003) Recent precipitation trends in Hungary in the context of larger scale climatic changes. Nat Hazards 29:255-271

Dutta S (2010) Multifractal properties of ECG patterns of patients suffering from congestive heart failure. J Stat Mech. doi:10. 1088/1742-5468/2010/12/P12021

Evertsz CJG, Mandelbrot BB (1992) Multifractal measures (Appendix B). In: Peitgen HO, Jurgens H, Saupe D (eds) Chaos and fractals. Springer, New York, pp 922-953

Feder J (1988) Fractals. Plenum Press, New York, pp 149-183

Gao H, Yang S (2009) A severe drought event in northern China in winter 2008-2009 and the possible influences of La Nina and Tibetan Plateau. J Geophys Res 114:D24104. doi:10.1029/ 2009JD012430

Govindan RB, Wilson JD, Preiß1 H, Eswaran H, Campbell JQ, Lowery CL (2007) Detrended fluctuation analysis of short datasets: an application to fetal cardiac data. Physica D 226:23-31

Grech D, Mazur Z (2004) Can one make any crash prediction in finance using the local Hurst exponent idea? Physica A 336:133-145

Halsey TC, Jensen MH, Kadanoff LP, Procaccia I, Shraiman BJ (1986) Fractal measures and their singularities: the characterization of strange sets. Phys Rev A 33:1141-1151

Hayes MJ, Svoboda MD, Wilhite DA, Vanyarkho OV (1999) Monitoring the 1996 drought using the standardized precipitation index. Bull Am Meteorol Soc 80:429-438

Hurst HE, Black RP, Simaika YM (1965) Long-term storage. Constable, London, p 145

Jafari GR, Pedram P, Hedayatifar L (2007) Long-term correlation and multifractality in Bach's inventions pitches. J Stat Mech. doi:10. 1088/1742-5468/2007/04/P04012

Jiang T, Zhang Q, Blender R, Fraedrich K (2005) Yangtze Delta floods and droughts of the last millennium: abrupt changes and long term memory. Theor Appl Climatol 82:131-141

Kantelhardt JW (2015) Fractal and multifractal time series. In: Meyers RA (ed) Encyclopedia of complexity and systems science. Springer, Berlin, pp 1-37. doi:10.1007/978-3-64227737-5_221-3

Kantelhardt JW, Zschiegner SA, Koscielny-Bunde E, Havlin S, Bunde A, Stanley HE (2002) Multifractal detrended fluctuation analysis of nonstationary time series. Physica A 316:87-114

Kantelhardt JW, Rybsk D, Zschiegner SA, Braun PE, Livina V, Bunde A, Havlin S (2003) Multifractality of river runoff and precipitation: comparison of fluctuation analysis and wavelet methods. Physica A 330:240-245

Kavasseri RG, Nagarajan R (2005) A multifractal description of wind speed records. Chaos Solitons Fract 24:165-173

Kimiagar S, Sadegh MM, Khorram S, Sobhanian S, Reza Rahimi Tabar M (2009) Fractal analysis of discharge current fluctuations. J Stat Mech. doi:10.1088/1742-5468/2009/03/P03020

Komuscu AU (1999) Using the SPI to analyze spatial and temporal patterns of drought in Turkey. Drought Netw News 11:7-13 
Koscielny-Bunde E, Kantelhardt JW, Braun P, Bunde A, Havlin S (2006) Long-term persistence and multifractality of river runoff records: detrended fluctuation studies. J Hydrol 322:120-137. doi:10.1016/j.jhydrol.2005.03.004

Lana X, Serra C, Burgueno A (2001) Patterns of monthly rainfall shortage and excess in terms of the standardized precipitation index for Catalonia (NE Spain). Int J Climatol 21:1669-1691

Lana X, Martínez MD, Serra C, Burgueño A (2010) Complex behavior and predictability of the European dry spell regimes. Nonlinear Proc Geophys 17:449-512

Li B, Su H, Chen F, Wu J, Qi J (2013) The changing characteristics of drought in China from 1982 to 2005. Nat Hazards 68:723-743

Lim G, Kim SY, Lee H, Kim K, Lee DI (2007) Multifractal detrended fluctuation analysis of derivative and spot markets. Physica A 386:259-266

Lloyd-Hughes B, Saunders MA (2002) Seasonal prediction of European spring precipitation from El Niño-southern oscillation and local sea-surface temperatures. Int J Climatol 22:1-14

Lorenz EN (1969a) Atmospheric predictability as revealed by naturally occurring analogues. J Atmos Sci 26:636-646

Lorenz EN (1969b) Three approaches to atmospheric predictability. Bull Am Meteorol Soc 50:345-349

Ma ZG, Fu CB (2006) Some evidence of drying trend over northern China from 1951 to 2004. Chin Sci Bull 51:2913-2925

Makowiec D, Galaska R, Dudkowska A, Rynkiewicz A, Zwierz M (2006) Long-term dependencies in heart rate signals-revisited. Physica A 369:632-644

Makowiec D, Rynkiewicz A, Galaska R, Wdowczyk-Szulc J, Arczynska-Buchowiecka M (2011) Reading multifractal spectra: aging by multifractal analysis of heart rate. Europhys Lett 94:68005

Mali P, Manna SK, Halda PK, Mukhopadhyay A, Singh G (2017) Detrended analysis of shower track distribution in nucleus-nucleus interactions at CERN SPS energy. Chaos Solitons Fract 94:86-94

Martínez MD, Lana X, Burgueño A, Serra C (2007) Lacunarity, predictability and predictive instability of the daily precipitation regime in the Iberian Peninsula. Nonlinear Proc Geophys 14:109-121

Martínez MD, Lana X, Burgueño A, Serra C (2010) Predictability of the monthly North Atlantic oscillation index based on dynamic systems theory. Nonlinear Proc Geophys 17:93-101

Matia K, Ashkenazy Y, Stanley HE (2003) Multifractal properties of price fluctuations of stocks and commodities. Europhys Lett 61:422-428

McKee TB, Doesken NJ, Kleist J (1993) The relationship of drought frequency and duration to time scales. In: Preprints. 8th conference on applied climatology, vol 17-22, pp 179-184

Mensia W, Tiwari AK, Yoon S-M (2017) Global financial crisis and weak-form efficiency of Islamic sectoral stock markets: an MFDFA analysis. Physica A 471:135-146

Min SK, Kwon WT, Park EH, Choi Y (2003) Spatial and temporal comparisons of droughts over Korea with East Asia. Int $\mathrm{J}$ Climatol 23:223-233

Moreira EE, Coelho CA, Paulo AA, Pereira SL, Mexia TJ (2008) SPIbased drought category prediction using loglinear models. J Hydrol 354:116-130

Movahed MS, Jafari GR, Ghasemi F, Rahvar S, Reza Rahimi Tabar M (2006) Multifractal detrended fluctuation analysis of sunspot time series. J Stat Mech 2:P02003

Niu M, Wang F, Liang Q, Yu G, Yu Z (2008) Multifractal detrended fluctuation analysis of pressure fluctuation signals in an impinging entrained-flow gasifier. Chem Eng J 136:364-372. doi:10. 1016/j.cej.2007.04.022

Norouzzadeh P, Rahmani B (2006a) A multifractal detrended fluctuation description of Iranian rial-US dollar exchange rate. Physica A 367:328-336
Norouzzadeh P, Rahmani B (2006b) Detrended fluctuation description of Iranian rial-Us dollar exchange rate. Physica A 367:328-336

Norouzzadeh P, Dullaert W, Rahmani B (2007) Anti-correlation and multifractal features of Spain electricity spot market. Physica A 380:333-342

Oh G, Eom C, Havlin S, Jung WS, Wang F, Stanley HE, Kim S (2012) A multifractal analysis of Asian foreign exchange markets. Eur Phys J B 85:214

Oswiecimka P, Kwapien J, Drozdz S (2005) Multifractality in the stock market: price increments versus waiting times. Physica A 347:626-638

Palmer WC (1965) Meteorological drought. Research paper, 45. U.S. Weather Bureau, Washington, DC, p 58

Pedram P, Jafari GR (2008) Mona lisa: the stochastic view and fractality in color space. Int J Mod Phys C 19:855-866. doi:10. 1142/S0129183108012558

Peng CK, Buldyrev SV, Simons M, Stanley HE, Goldberger AL (1994) Mosaic organization of DNA nucleotides. Phys Rev E 49:1685-1689

Qian WH, Lin X (2005) Regional trends in recent precipitation indices in China. Meteorol Atmos Phys 90:193-207

Qian WH, Ding T, Hu HR, Lin X, Qin AM (2009) An overview of dry-wet climate variability among monsoon-westerly regions and the monsoon northernmost marginal active zone in China. Adv Atmos Sci 26:630-641

Qiu J (2010) China drought highlights future climate threats. Nature 465:142-143

Seiler RA, Hayes M, Bressan L (2002) Using the standardized precipitation index for food risk monitoring. Int $\mathrm{J}$ Climatol 22:1365-1376

Silva Y, Takahashi K, Chavez R (2007) Dry and wet rainy seasons in the Mantaro River basin (Central Peruvian Andes). Adv Geosci 14:1-4

Small M, Tse CK (2003) Detecting determinism in time series: the method of surrogate data. IEEE Trans Circuits Syst I: Fundam Theory Appl 50:663-672

Song LC, Deng ZL, Dong AX (2003) Drought. China Meteorological Press, Beijing, p 162

Sun X, Chen HP, Yuan YZ, Wu ZQ (2001) Predictability of multifractal analysis of Hang Seng Stock index in Hong Kong. Physica A 301:473-482

Tatli H (2014) Statistical complexity in daily precipitation of NCEP/ NCAR reanalysis over the Mediterranean Basin. Int J Climatol 34:155-161

Tatli H (2015) Detecting persistence of meteorological drought via Hurst exponent. Meteorol Appl 22:763-769

Telesca L, Colangelo G, Lapenna V, Macchiato M (2004) Fluctuation dynamics in geoelectrical data: an investigation by using multifractal detrended fluctuation analysis. Phys Lett A 332:398-404

Tokinaga S (2000) The analysis of the economy models based on the complex system. Kyushu University Press, Fukuoka

Tsonis A (1992) Chaos: from theory to applications. Plenum, New York

Turcotte DL (1997) Fractal and chaos in geology and geophysics, 2nd edn. Cambridge University Press, New York, p 398

Van-Rooy MP (1965) A rainfall anomaly index (RAI) independent of time and space. Notos 14:43-48

Vicente-Serrano SM, Begueria S, Lopez-Moreno JI, Angulo M, El Kenawy A (2010) A new global 0.5 degrees gridded dataset (1901-2006) of a multiscalar drought index: comparison with current drought index datasets based on the palmer drought severity index. J Hydro Meteorol 11:1033-1043

Wang YD, Wu CF, Pan ZY (2011a) Multifractal detrending moving average analysis on the US Dollar exchange rates. Physica A 390:3512-3523 
Wang AH, Lettenmaier DP, Sheffield J (2011b) Soil moisture drought in China, 1950-2006. J Clim 24:3257-3271

Wei Y, Huang D (2005) Multifractal analysis of SSEC in Chinese stock market: a different empirical results from the Heng Seng index. Physica A 355:497-508

Wei Y, Wang P (2008) Forecasting volatility of SSEC in Chinese stock market using multifractal analysis. Physica A 387:1585-1592

Weng HY, Wu GX, Liu YM, Behera SK, Yamagata T (2011) Anomalous summer climate in China influenced by the tropical Indo-Pacific Oceans. Clim Dyn 36:769-782

Wu H, Hayes MJ, Wilhite DA, Svoboda MD (2005) The effect of the length of record on the standardized precipitation index calculation. Int J Climatol 25:505-520

Xie S, Bao Z (2004) Fractal and multifractal properties of geochemical fields. Math Geol 36:847-864

Yu ZG, Leung Y, Chen YD, Zhang Q, Anh V, Zhou Y (2014) Multifractal analyses of daily rainfall time series in Pearl River basin of China. Physica A 405:193-202. doi:10.1016/j.physa. 2014.02.047

Yuan Y, Zhuang XT, Jin X (2009) Measuring multifractality of stock price fluctuation using multifractal detrended fluctuation analysis. Physica A 388:2189-2197

Zhai PM, Zhang XB, Wan H, Pan XH (2005) Trends in total precipitation and frequency of daily precipitation extremes over China. J Clim 18:1096-1108

Zhang Q, Xu CY, David Chen YQ, Gemmer M, Yu ZG (2008) Multifractal detrended fluctuation analysis of streamflow series of the Yangtze River basin, China. Hydrol Process 22:4997-5003. doi:10.1002/hyp.7119

Zhang Q, Xu CY, Zhang ZX (2009a) Observed changes of drought/ wetness episodes in the Pearl River basin, China, using the standardized precipitation index and aridity index. Theor Appl Climatol 98:89-99

Zhang Q, Chong YX, Yu ZG, Liu CL, David Chen YQ (2009b) Multifractal analysis of streamflow records of the East River basin (Pearl River), China. Physica A 388:927-934. doi:10.1016/ j.physa.2008.11.025

Zhang Q, Yu ZG, Xu CY, Anh V (2010a) Multifractal analysis of measure representation of flood/drought grade series in the Yangtze Delta, China, during the past millennium and their fractal model simulation. Int J Climatol 30:450-457

Zhang Q, Jiang T, Chen YD, Chen XH (2010b) Changing properties of hydrological extremes in south China: natural variations or human influences? Hydrol Process 24:1421-1432

Zhang Q, Zhou Y, Singh Vijay P, David Chen YQ (2011) Comparison of detrending methods for fluctuation analysis in hydrology. J Hydrol 400:121-132. doi:10.1016/j.jhydrol.2011.01.032

Zhang Q, Zhou Y, Singh Vijay P (2014) Detrending methods for fluctuation analysis in hydrology: amendments and comparisons of methodologies. Hydrol Process 28:53-712. doi:10.1002/hyp. 9637

Zhou W, Chan JCL, Chen W, Ling J, Pinto JG, Shao Y (2009) Synoptic-scale controls of persistent low temperature and icy weather over Southern China in January 2008. Mon Weather Rev 137:3978-3991. doi:10.1175/2009MWR2952.1 